\title{
The role of shear deformation component in pressure welding samples of dissimilar cast and wrought nickel-based alloys
}

\author{
A.Kh. Akhunova ${ }^{1, \dagger}$, E.V. Galieva ${ }^{1}$, A.A. Drozdov ${ }^{2}$, E.G. Arginbava ${ }^{3}$, S.V. Dmitriev ${ }^{1,4}$, \\ R.Ya. Lutfullin ${ }^{1}$ \\ †akhunova_a@mail.ru \\ ${ }^{1}$ Institute for metals superplasticity problems of RAS, 39 Khalturin str., 450001, Ufa, Russia \\ ${ }^{2}$ Institute of Metallurgy and Material Science of RAS, 49 Leninsky pr., 119991, Moscow, Russia \\ 3"VIAM" FSUE, RF SRC. 17 Radio Str., 105005, Moscow, Russia \\ ${ }^{4}$ National Research Tomsk State University, Lenin Ave, 36, Tomsk 634050, Russia
}

\begin{abstract}
A material plastic flow finite-element modeling during pressure welding (PW) of two-element and three-element samples was carried out for dissimilar wrought (EP975) and cast intermetallic (VKNA-type) alloys at $1125^{\circ} \mathrm{C}$ and $10^{-4} \mathrm{~s}^{-1}$ initial strain rate. The finite-element modeling was performed in a two-dimensional set-up (plane strain). In the two-element samples case, the top element was ultrafine grained EP975 and the bottom one was VKNA-25. The top and bottom elements of the threeelement samples were made from VKNA-25 while the intermediate element was ultrafine-grained EP975. The geometry in the $X$ and $Y$ directions were 40.5 and $5 \mathrm{~mm}$ for the EP975 samples and 40.5 and $3 \mathrm{~mm}$ for the VKNA-25 samples respectively. Experiments on PW intermetallic $\mathrm{Ni}_{3} \mathrm{Al}$ alloy and heat-resistant nickel alloy EP975 with ultrafine-grained (UFG) structure were carried out. Samples were parallelepipeds of size $10 \times 40 \times 3 \mathrm{~mm}^{3}$ and $10 \times 40 \times 5 \mathrm{~mm}^{3}$ respectively. PW was carried out at $1125^{\circ} \mathrm{C}$. Several combinations of welded sample surfaces with flat and grooved relief were considered. The relief made on both welded surfaces was equivalent to increase of friction between them. This results in a shear deformation reduction near the contact surfaces and degrades the welding quality as compared to the flat surfaces conventional welding. Relief applied only to one of the elements being welded results in a more efficient welding process since active deformation zones are created in the central zone, where in the other samples stagnant zones are observed. There are no deformation peculiarities in PW of the three-element samples, compared to two-element sample.
\end{abstract}

\section{Роль сдвиговой компоненты деформации при сварке дав- лением образцов из разнородных литых и деформируемых сплавов на основе никеля}

\author{
Ахунова А.Х. ${ }^{1 \dagger}$, Галиева Э.В. ${ }^{1}$, Дроздов А.А. ${ }^{2}$, Аргинбаева Э.Г. ${ }^{3}$, Дмитриев С.В. ${ }^{1,4}$, \\ Лутфуллин Р.Я. ${ }^{1}$ \\ †akhunova_a@mail.ru \\ ${ }^{1}$ Институт проблем сверхпластичности металлов РАН, ул. Халтурина 39, 450001, Уфа, Россия \\ ${ }^{2}$ Институт металлургии и материаловедения им. А.А. Байкова, г. Москва, Россия \\ ${ }^{3}$ ФУП ГНЦ «ВИАМ», г. Москва, Россия
}

${ }^{4}$ Национальный исследовательский Томский государственный университет, просп. Ленина 36, 634050 Томск, Россия

Проведено конечно-элементное моделирование пластического течения материала при сварке давлением двух- и трехэлементных образцов из разнородных деформируемых (ЭП975) и литых интерметаллидных (типа ВКНА) сплавов в температурно-скоростных условиях сверхпластической деформации сплава ЭП975 с ультрамелкозернистой (УМЗ) структурой. Моделирование проводили в двумерной постановке (плоское деформированное состояние). В двухэлементном образце верхний элемент был изготовлен из жаропрочного сплава ЭП975 в УМЗ состоянии и нижний - 
из интерметаллидного сплава ВКНА-25. В трехэлементном образце верхний и нижний элементы были изготовлены из интерметаллидного сплава ВКНА-25, промежуточный - из жаропрочного сплава ЭП975 в УМЗ состоянии. Геометрические размеры элементов из сплава ЭП975 и ВКНА- 25 по осям $X$ и $Y$ составляли 40,5×5 мм² и 40,5×3 мм², соответственно. Проведено физическое моделирование сварки давлением (СД) образцов из интереметаллидного сплава на основе $\mathrm{Ni}_{3} \mathrm{Al}$ и жаропрочного никелевого сплава ЭП975 с УМЗ структурой, образцы представляли собой параллелепипеды размером $10 \times 40 \times 3 \mathrm{mм}^{3}$ и $10 \times 40 \times 5 \mathrm{~mm}^{3}$ соответственно. СД осуществляли при температуре $1125^{\circ} \mathrm{C}$. Рассмотрено несколько вариантов сочетаний свариваемых поверхностей образцов. Показано, что вариант сварки при, при котором рельеф наносится на обе свариваемые поверхности, эквивалентен повышению трения между контактными поверхностями. Это приводит к значительному снижению сдвиговой деформации вблизи контактной поверхности, и ухудшает качество сварки по сравнению с традиционной сваркой плоских поверхностей. Более эффективным вариантом сварки является сварка образцов с нанесением рельефа только на один свариваемый элемент, в этом случае возникают зоны активной деформации в центральной области, где в других сочетаниях появляются застойные зоны. Установлено, в случае трехэлементного образца каких-либо особенностей деформации не возникает, и результаты моделирования для двухэлементного образца, можно использовать для анализа напряженно-деформированного состояния на соответствующих контактных поверхностях трехэлементного образца.

Ключевые слова: конечно-элементное моделирование, сварка давлением, рельеф, жаропрочный никелевый сплав, интерметаллидные сплавы на основе $\mathrm{Ni}_{3} \mathrm{Al}$.

\section{1. Введение}

Для получения неразъемных соединений диска с лопатками весьма перспективным является процесс сварки давлением (СД), в частности, в условиях сверхпластичности (СП) [1,2]. Поскольку к дисковой и лопаточной частям блиска предъявляются различные требования по прочностным и жаропрочным свойствам, то для их создания целесообразно использовать жаропрочные литейные интерметаллидные сплавы, из которых могут быть изготовлены лопатка [3], а для изготовления дисковой части, работающей при умеренных температурах $\left(\leq 850^{\circ} \mathrm{C}\right)$, целесообразно применять высокожаропрочные деформируемые сплавы [4,5], например, сплав ЭП975.

Работоспособность деталей типа «блиск» определяется, главным образом, качеством соединения замковой части лопатки с диском, поскольку наличие дефектов в таких местах может привести не только к разрушению этой детали, но и к разрушению всего двигателя. Поэтому достижение высокого качества сварного соединения в таких биметаллических деталях является актуальной задачей.

При СД твердофазное соединение формируется в результате пластической деформации и диффузионных процессов в зоне соединения, которые в значительной степени определяются физико-механическими свойствами свариваемых разнородных материалов [1-3]. Условия протекания деформации приконтактных объемов, такие как степень и скорость деформации, время сварки, структурные превращения при деформации и нагреве влияют на качество и эксплуатационные свойства сварного соединения [6-8]. При этом характер микроструктуры также оказывает значительное влияние на свойства материала $[9,10]$. В частности, высоколегированные сплавы на никелевой основе, является гетерофазным, вследствие чего в исходном крупнозернистом состоянии имеют низкую технологическую пластичность. Одним из наиболее перспективных путей повышения технологической пластичности жаропрочных никелевых сплавов является перевод их в сверхпластичное состояние $[11,12]$. Тем не менее, при соединении деталей с большими контактными поверхностями нередко образуются застойные зоны, где напряженное состояние близко к гидростатическому давлению и пластическая деформация отсутствует, так как для ее протекания необходимо наличие сдвигающих усилий. Одним из решений данной проблемы является нанесение рельефа (например, проточек) на свариваемых поверхностях с целью обеспечения локальной пластической деформации в зоне соединения. Для выявления влияния рельефа на процесс сварки целесообразно использовать компьютерное моделирование, которое позволяет проанализировать все аспекты данного технологического процесса [13-15]. Во-первых, моделирование дает возможность судить о качестве полученного соединения в образце, в частности, все приконтактные объемы должны быть заполнены. Во-вторых, по результатам моделирования можно определить геометрические параметры образца, такие как размер, форму и местоположение проточек. В-третьих, в результате моделирования определяются временные характеристики процесса - скорость деформирования и время сварки. Например, в работе [16] посредством компьютерного моделирования установлены закономерности формирования напряженно-деформированного состояния металла в зоне соединения при диффузионной сварке титана с низкоинтенсивным силовым воздействием. Также получено выражение, позволяющее прогнозировать кинетику развития физического контакта при диффузионной сварке в зависимости от технологических параметров процесса.

В данной работе методами конечно-элементного моделирования было исследовано влияние рельефа на свариваемой поверхности образца из интерметаллидного сплава на качество готового твердофазного соединения.

\section{2. Методика компьютерного мо- делирования и эксперимента}

Компьютерное моделирование. Компьютерное моделирование проводили в двумерной постановке (плоское деформированное состояние) с помощью пакета прикладных программ DEFORM-2D. Моделировалась сварка давлением двух типов образцов (рис. 1). 
Образец I состоял из двух элементов: верхнего элемента из жаропрочного сплава ЭП975 в УМЗ состоянии и нижнего - из интерметаллидного сплава ВКНА-25 (рис. 1). Образец II состоял из трёх элементов: верхнего и нижнего элементов из интерметаллидного сплава ВКНА-4У и промежуточного - из жаропрочного сплава ЭП975 в УМЗ состоянии (рис. 2). Геометрические размеры элементов из сплава ЭП975 и ВКНА-25 по осям $X$ и $Y$ составляли $40,5 \times 5$ мм $^{2}$ и $40,5 \times 3$ мм $^{2}$, соответственно.

Отметим, что трехэлементный образец II был введен в рассмотрение, так как он наиболее близок к условиям сварки давлением лопатки и диска как конструктивно, так и по напряженно-деформированному состоянию (НДС), возникающему в свариваемых деталях.

Рассматривали задачи, при которых на поверхность свариваемого элемента образца из сплава ВКНА-25, имитирующего поведение литого лопаточного материала, наносились проточки, форма которых составлена из сегментов окружности радиуса 0,5 мм и углом раствора $90^{\circ}$. Изучали несколько видов сочетаний свариваемых поверхностей:

- все свариваемые поверхности плоские (рис. 1а и рис. 2a);

- все свариваемые поверхности имели рельеф (рис. $1 \mathrm{~b}$ и рис. $2 \mathrm{~b}$ );

- элементы из сплава ЭП975 имели плоскую свариваемую поверхность, рельеф наносился только на поверхности элемента из сплава ВКНА-25 (рис. 1с и рис. 2c).

Сварку давлением моделировали при температуре $1125^{\circ} \mathrm{C}$ и начальной скорости деформации $10^{-4} \mathrm{c}^{-1}$. Отметим, что сплав ЭП975 в ультрамелкозернистом (УМЗ) состоянии является намного более пластичным, чем литой интерметаллидный сплав ВКНА-25, который при выбранных условиях деформации практически не деформируется.

Сварка давлением. СД осуществляли по схеме одноосного сжатия в условиях СП никелевого сплава ЭП975 при температуре $1125^{\circ} \mathrm{C}$. Было рассмотрено две задачи: 1) все свариваемые поверхности были плоские; 2) на образцы из интереметаллидного сплава на основе $\mathrm{Ni}_{3} \mathrm{Al}$ был нанесен рельеф на электроискровом станке в виде сегментов окружностей с радиусом окружности 0,5 мм, при этом образцы из жаропрочного никелевого сплава ЭП975 были плоские, геометрические размеры образцов составляли $10 \times 40 \times 5 \mathrm{Mм}^{2}$ и $10 \times 40 \times 5 \mathrm{Mм}^{2}$ соответственно. Прочность соединения оценивали по результатам механических испытаний на сдвиг при комнатной температуре.

\section{3. Результаты и обсуждение}

По результатам моделирования можно заключить, что при СД трехэлементного образца каких-либо особенностей деформации не возникает, и картины распределения интенсивности деформаций и сдвиговой деформации трехэлементного образца для всех рассматриваемых сочетаний контактных поверхностей эквивалентны картинам распределения двухэлементного образца. На рис. 3 и 4 приведены результаты моделирования сварки в виде картин распределения интен- сивности деформаций для рассматриваемых сочетаний поверхности для двухэлементного образца I и трехэлементного образца II, соответственно.

Известно [12], что при сварке плоских поверхностей в центральной части образца образуются так называемые застойные зоны деформации, которые хорошо видны как двух-, так и трехэлементном образцах I и II (рис. 3а и 4а). Это объясняется тем, что в центральной

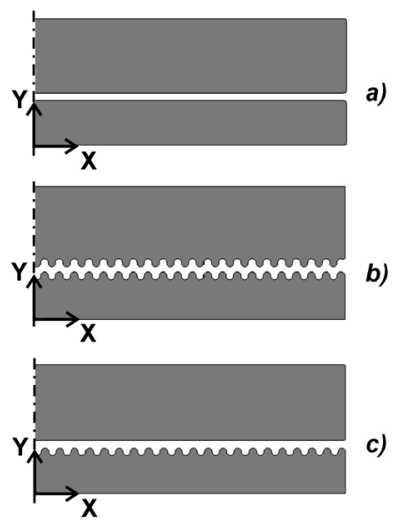

Рис. 1. Конечно-элементная модель свариваемых образцов типа I: обе свариваемые поверхности плоские (а), обе свариваемые поверхности имеют рельеф (b), рельеф на свариваемой поверхности имеет только нижний элемент из сплава ВКНА25 , свариваемая поверхность верхнего элемента из сплава ЭП975 - плоская (c).

Fig. 1. Finite-element model of a solid-phase pressure-welded specimen I type: sample I - both pressure welded surfaces are flat (a), only the bottom element from VKNA-25 alloy has a relief 0.5 $\mathrm{mm}$ high, welded surface of the top element from EP975 alloy has a flat relief (b).

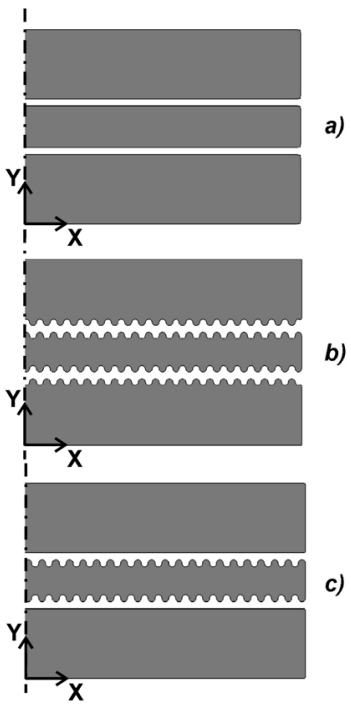

Рис. 2. Конечно-элементная модель свариваемых образцов типа II: все свариваемые поверхности плоские (а), все свариваемые поверхности имеют рельеф (b), рельеф на свариваемых поверхностях имеет только промежуточный элемент из сплава ВКНА-25, свариваемые поверхности верхнего и нижнего элементов из сплава ЭП975 - плоские (c).

Fig. 2. Finite-element model of a solid-phase pressure-welded specimen II type: all pressure welded surfaces are flat (a), all pressure welded surfaces have complementary reliefs $0.5 \mathrm{~mm}$ high (b), only the intermediate element from VKNA-25 alloy has a relief $0.5 \mathrm{~mm}$ high, welded surface of the top and bottom elements from EP975 alloy have a flat relief. 
части образца гидростатическое давление высоко, пластическое течение материала вдоль свариваемых поверхностей практически отсутствует и сдвиговая компонента деформации очень мала, что приводит к ухудшению качества сварки. Одним из способов избежать этого, является нанесение рельефа на свариваемые поверхности, поскольку возникновение участков локальной деформа-
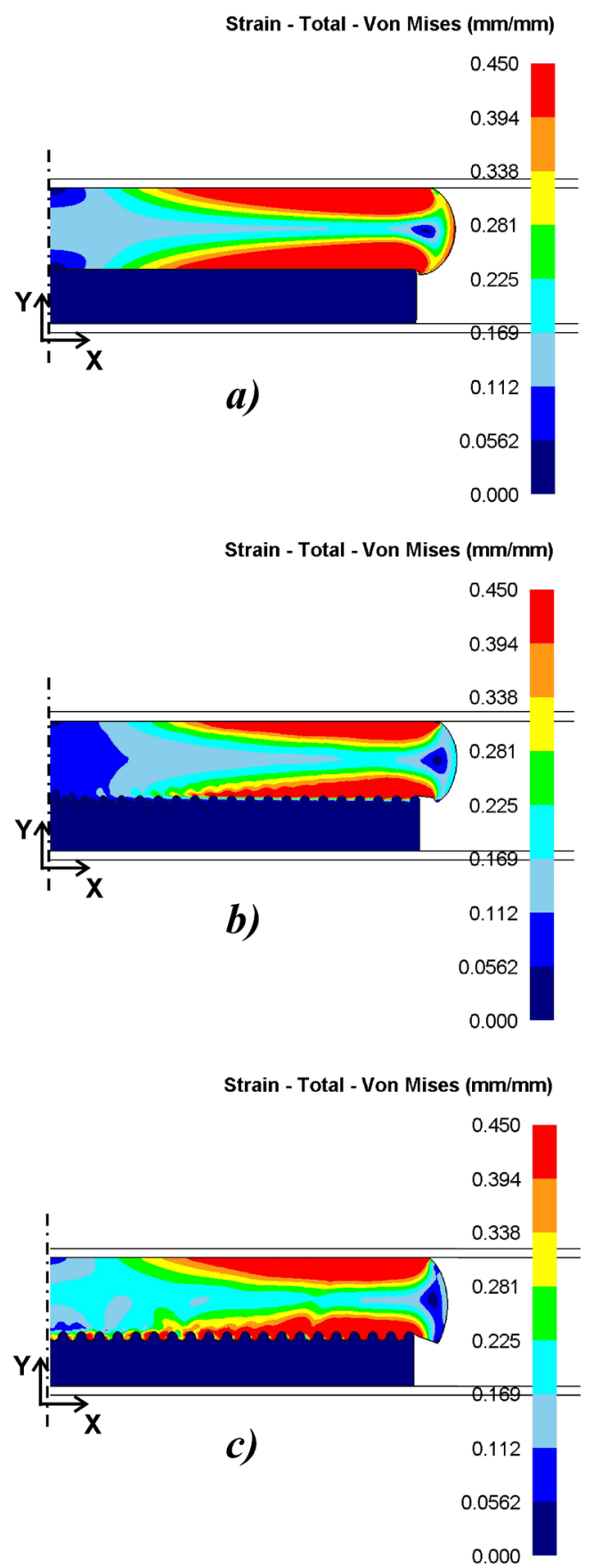

Pис. 3. (Color online) Распределение интенсивности деформаций для образца I: обе свариваемые поверхности плоские (a), обе свариваемые поверхности имеют рельеф (b), рельеф только на свариваемой поверхности нижнего элемента, свариваемая поверхность верхнего элемента - плоская (c).

Fig. 3. (Color online) Strain intensity distribution patterns for samples I type: sample I - both pressure welded surfaces are flat (a), sample II - both pressure welded surfaces have relief (b), only the bottom element has a relief (c). ции улучшает качество сварного шва. Однако, в случае нанесения рельефа на обе свариваемые поверхности деформации, в случае взаимной комплементарности рельефов, картина распределения деформаций остается в основном прежней (рис. $3 \mathrm{~b}$ и $4 \mathrm{~b}$ ). В данном случае, сочетание таких рельефных поверхностей равносильно созданию большого трения - движение материала вдоль

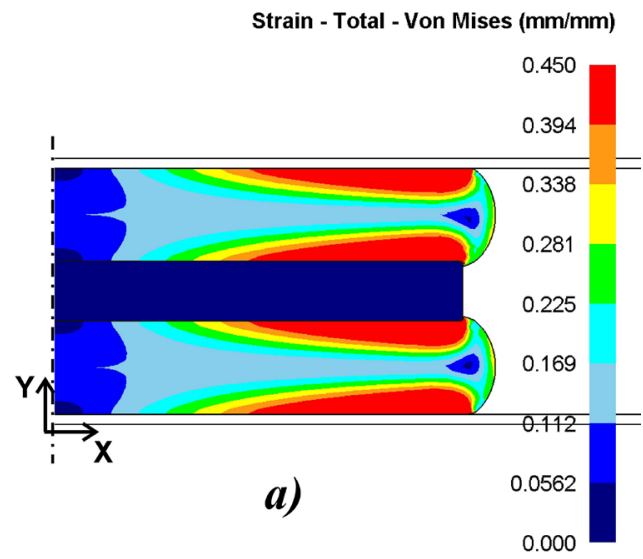

Strain - Total - Von Mises $(\mathrm{mm} / \mathrm{mm})$

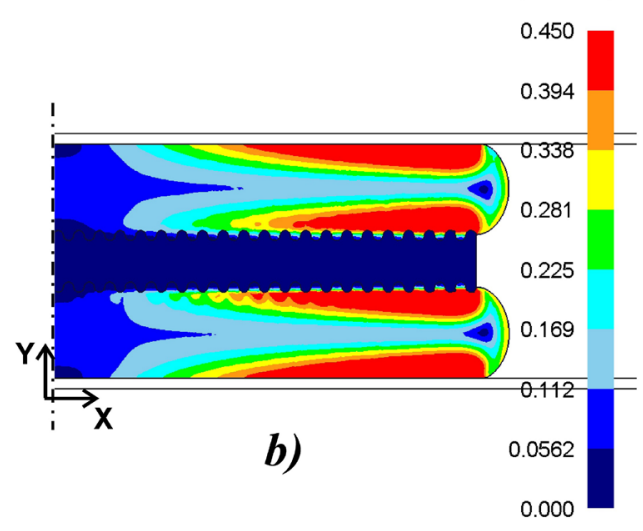

Strain - Total - Von Mises $(\mathrm{mm} / \mathrm{mm})$

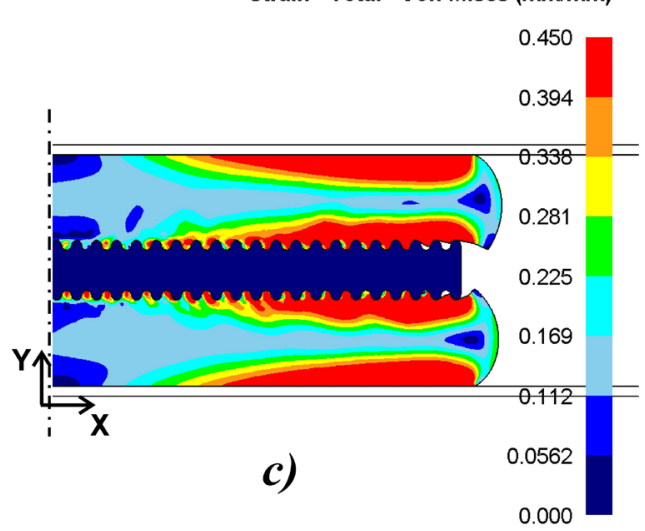

Рис. 4. (Color online) Распределение интенсивности деформаций для образца II: все свариваемые поверхности плоские (а), все свариваемые поверхности имеют рельеф (b), рельеф на свариваемых поверхностях имеет только промежуточный элемент, свариваемые поверхности верхнего и нижнего элементов - плоские (c).

Fig. 4. (Color online) Strain intensity distribution patterns for samples I type: sample II - all pressure welded surfaces are flat (a); all pressure welded surfaces have relief (b); only the bottom element has a relief; only the intermediate element has relief, the bottom and top elements are flat (c). 
поверхности сварки уменьшается, что и приводит к значительному снижению сдвиговой компоненты деформации вблизи поверхностей касания.

Отметим, что начальная контактная площадь в образцах с взаимно комплементарным рельефом больше, чем в образцах с плоскими сварными поверхностями, однако со временем она и в том и в другом случае практически не увеличивается. Это создает неблагоприятные условия для пластической деформации, что приводит к образованию зон затрудненной деформации.

При сварке плоской поверхности с рельефной поверхностью (рис. 3с и 4c) более пластичный материал верхнего элемента заполняет проточки нижнего элемента. За счёт деформации плоской поверхности происходит не только увеличение площади контакта, но и увеличение сдвиговой компоненты деформации. Это ведет к возникновению локальных очагов деформации по поверхности сварки, которые, как известно [12,13], улучшают качество сварного шва.

Результаты моделирования показали, что при сварке плоской и рельефной поверхности (рис. 1с и 2с) возникают области со значительной сдвиговой деформацией по всей зоне соединения, в отличие от образцов с плоскими (рис. 1a и $2 \mathrm{a}$ ) и взаимно рельефными (рис. $1 \mathrm{~b}$ и $2 \mathrm{~b}$ ) поверхностями, где в центре образцов хорошо видны застойные зоны деформации. Исходя из этого, можно заключить, что при сварке образцов с плоской и рельефной поверхностностями в его центральной части можно наблюдать лучшее качество сварки в отличие от образцов с другими сочетаниями поверхностей. Полученные результаты коррелируют с экспериментальными данными. По результатам физического моделирования показано, что наличие рельефа благоприятно влияет на локализацию деформации, снижается величина относительной протяженности пор (рис. 5) и, как следствие, повышает качество соединения. Об этом свидетельствуют результаты механических испытаний на сдвиг, которые показали, что при наличии рельефа разрушение происходит не по поверхности соединения, а по основному материалу, в отличие от образцов с плоской поверхностью соединения, в которых разрушение идет по поверхности соединения.

Таким образом, из анализа данных, полученных по результатам моделирования можно сделать заключение, что более эффективным методом подготовки свариваемых поверхностей является вариант, при котором проточки наносятся только на одну свариваемую поверхность.

\section{4. Выводы}

На основании проведенного расчетного моделирования можно отметить следующие основные результаты:

1) Проведено конечно-элементное моделирование пластического течения материала в зоне контакта двух и трех поверхностей, свариваемых в твердой фазе при температуре $1125^{\circ} \mathrm{C}$ и начальной скорости деформации $10^{-4} \mathrm{c}^{-1}$.

2) Установлено, что при сварке давлением трехэлементного образца каких-либо особенностей деформа- ции не возникает, и результаты моделирования, полученные для двухэлементного образца, можно использовать для анализа напряженно-деформированного состояния на соответствующих контактных поверхностях трехэлементного образца.

3) Показано, что метод сварки давлением, при условии, что рельеф наносится на обе свариваемые поверхности, эквивалентен повышению трения между контактными поверхностями. Это приводит к значительному снижению сдвиговой деформации вблизи контактной поверхности, и, как следствие, ухудшает качество сварки по сравнению с традиционной сваркой плоских поверхностей.

4) Более эффективным вариантом является сварка образцов с нанесением рельефа только на один свариваемый элемент, поскольку в этом случае возникают зоны активной деформации в центральной области, где в других образцах появляются застойные зоны.

Благодарность/Acknowledgements. А.Х. А. благодарит РФФИ за региональный грант РФФИ 14-08-97061 р_поволжье_а; Э.В. Г., В.А. В., Р.Я. Л., Э.Г. А. и А.А. Д. благодарят РФФИ за грант РФФИ № 13-08-12200; С.В. Д. благодарит грант РНФ № 16-12-10175.
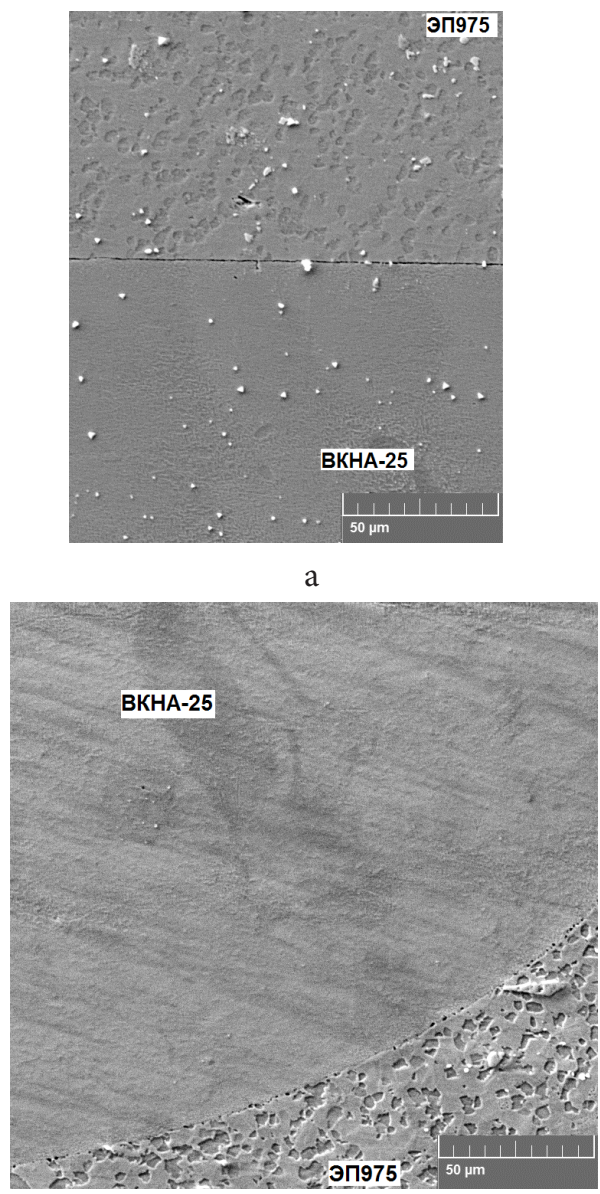

b

Рис.5. Микроструктура зоны ТФС ЭП975//ВКНА-25: (а) плоская поверхность соединения; (b) свариваемая поверхность сплава ВКНА-25 имеет рельеф.

Fig. 5. SPJ zone microstructure EP975//VKNA-25 (a) - all pressure welded surfaces are flat; (b) VKNA-25 welded surface has a relief. 


\section{Литература/References}

1. E. S. Karakozov. Soedinenie metallov v tverdoi phase. M.: Metallurgy, (1976) 224 p. (in Russian) [Э.С. Каракозов Соединение металлов в твердой фазе. М.: Металлургия, 1976, 224c]

2. R. Ya. Lutfullin. Letters on Materials. 1 (1), 59-64 (2011). (in Russian) [Р. Я. Лутфуллин. Письма о материалах. 1 (1), 59-64 (2011).]

3. K.B. Povarova, V.A. Valitov, S.V. Ovsepyan, A.A. Drozdov, O.A. Bazyleva, E.V. Valitova. Russian metallurgy. (9), $733-741$ (2014)

4. B.S. Lomberg, S.V. Ovsepyan, M.M. Bakradze, I. S. Mazalov. 80 years. Aviacionnye materialy i tekhnologii: yubil. nauchn.-techknich. sb. (prilozhenie $\mathrm{k}$ zhurnalu Aviacionnye materialy). M. VIAM, $52-57$ (2012). (in Russian) [Б. С. Ломберг, С. В. Овсепян, М. М. Бакрадзе, И. С. 80 лет. Авиационные материалы и технологии: юбил. науч.-технич. сб. (приложение к журналу «Авиационные материалы и технологии»)/под общ. ред. акад. РАН, проф. Е.Н. Каблова. - М.: Изд. ВИАМ, 2012. С. 52 - 57].

5. A. V. Logunov, Yu. N. Shmotin. Modern high temperature nickel alloys for disks of gas turbines. Moscow. Public corporation «Science and Technology». (2013) 256 p. (in Russian) [А.В. Логунов, Ю.Н. Шмотин. Наука и технология. (2013) 256 с.]

6. E.V. Valitova, A.Kh. Akhunova, V.A. Valitov, S. V. Dmitriev, R. Ya. Lutfullin, Letters on Materials. 4 (3), 190 - 194 (2014). (in Russian) [Э.В. Валитова, А.Х. Ахунова, В.А. Валитов, С.В. Дмитриев, Р.Я. Лутфуллин, М.Х. Мухаметрахимов. Письма о материалах. 4 (3), 190 - 194 (2014)]

7. A.Kh. Akhunova, S.V. Dmitriev, E.V. Galieva, V.A. Valitov. Fundamentalnye problem sovremennogo materialovedeniya. 12 (3), 289-292 (2015). (in Russian)
[А.Х. Ахунова, С.В. Дмитриев, Э.В. Галиева, В.А. Валитов. Фундаментальные проблемы современного материаловедения. 12 (3), 289 - 292 (2015).]

8. E. V.Valitova, R. Ya.Lutfullin, M. Kh. Mukhametrakhimov, V.A. Valitov, A. Kh. Akhunova, S. V. Dmitriev. Letters on Materials. 4 (4), 291 - 294 (2014).

9. I.I. Musabirov, R.R. Mulyukov, V.V. Koledov. IOP Conference Series: Materials Science and Engineering. 82, 012064-1-4 (2015)

10. I.I. Musabirov, I.Z. Sharipov, R.R. Mulyukov. Russian Physics Journal. 58 (6), 5-9 (2015)

11. V.A. Valitov. Tyazheloe mashinostroenie. (4), 23-28 (2007). (in Russian) [Валитов В.А. Тяжелое машиностроение. (4), 23 - 28 (2007).]

12. E. V.Valitova, M. Kh.Mukhametrakhimov, R. Ya.Lutfullin, V. A. Valitov. Perspectivnye materialy. (15), 35 - 39 (2013). (in Russian) [Э.В. Валитова, М.Х. Мухаметрахимов, Р. Я. Лутфуллин, В. А. Валитов. Перспективные материалы 15, 33 - 39 (2013).]

13. A.Kh. Akhunova, S.V. Dmitriev, E.V. Valitova, V.A. Valitov. Fundamentalnye problem sovremennogo materialovedeniya. 11 (2), 159 - 162 (2014). (in Russian) [А.Х. Ахунова, С.В. Дмитриев, Э.В. Валитова, В.А. Валитов. Фундаментальные проблемы современного материаловедения. 11 (2), 159 - 162 (2014).]

14. A.Kh. Akhunova, S.V. Dmitriev, V.A. Valitov, E. V. Valitova. Russian metallurgy (Metally) (11), 13-17 (2014). (in Russian) [А.Х. Ахунова, С.В. Дмитриев, В. А. Валитов, Э. В. Валитова Деформация и разрушение материалов. (11), 13 - 17 (2014).]

15. A.Kh. Akhunova, E.V. Valitova, S.V. Dmitriev, V.A. Valitov, R. Ya. Lutfullin Welding International. 30 (6), $488-491$ (2016).

16. A. K. Bulkov, V. V. Peshkov, V. R. Petrenko, D. N. Balbekov, A. I. Stryguin. Welding International. 28 (3), 222-227 (2014). 